\title{
Mesenchymal Stem Cells and the Origin of Ewing's Sarcoma
}

\author{
Patrick P. Lin, ${ }^{1}$ Yongxing Wang, ${ }^{1}$ and Guillermina Lozano ${ }^{2}$ \\ ${ }^{1}$ Department of Orthopaedic Oncology (Unit 1448), University of Texas MD Anderson Cancer Center, 1515 Holcombe Boulevard, \\ Houston, TX 77030, USA \\ ${ }^{2}$ Department of Genetics (Unit 1010), University of Texas MD Anderson Cancer Center, 1515 Holcombe Boulevard, \\ Houston, TX 77030, USA
}

Correspondence should be addressed to Patrick P. Lin, plin@mdanderson.org

Received 14 July 2010; Accepted 6 September 2010

Academic Editor: Cyril Fisher

Copyright (C) 2011 Patrick P. Lin et al. This is an open access article distributed under the Creative Commons Attribution License, which permits unrestricted use, distribution, and reproduction in any medium, provided the original work is properly cited.

The origin of Ewing's sarcoma is a subject of much debate. Once thought to be derived from primitive neuroectodermal cells, many now believe it to arise from a mesenchymal stem cell (MSC). Expression of the EWS-FLI1 fusion gene in MSCs changes cell morphology to resemble Ewing's sarcoma and induces expression of neuroectodermal markers. In murine cells, transformation to sarcomas can occur. In knockdown experiments, Ewing's sarcoma cells develop characteristics of MSCs and the ability to differentiate into mesodermal lineages. However, it cannot be concluded that MSCs are the cell of origin. The concept of an MSC still needs to be rigorously defined, and there may be different subpopulations of mesenchymal pluripotential cells. Furthermore, EWS-FLI1 by itself does not transform human cells, and cooperating mutations appear to be necessary. Therefore, while it is possible that Ewing's sarcoma may originate from a primitive mesenchymal cell, the idea needs to be refined further.

\section{Introduction}

Ewing's sarcoma is a rare malignancy primarily affecting children and adolescents. It arises mainly in bone and less commonly in soft tissues. The poorly differentiated tumors are aggressive and metastasize early to lung, bone marrow, and other tissues [1-4]. In all cases of the disease, there is a characteristic reciprocal chromosomal translocation, which leads to an in-frame fusion between the EWS gene and one of the ETS family gene members $[5,6]$. In approximately $85 \%$ of cases, the EWS gene is combined with the ETS gene FLI1 in a $\mathrm{t}(11 ; 22)$ translocation. In about $10 \%$ of cases, EWS is fused to ERG, which has a high degree of homology with FLI1 in the carboxyl terminus. In very rare cases, EWS is fused to other ETS family genes such as FEV and ETV1 [1,7]. The EWS-ETS fusion gene appears to be critically important for maintaining the tumor phenotype of the disease. Ewing's sarcoma cells with EWS-FLI1 knockdown by siRNA exhibit decreased cell proliferation, and tumor xenografts regress in mice [8, 9].

\section{Mechanism for EWS-FLI1 in Tumorigenesis}

The mechanism by which EWS-FLI1 contributes to tumorigenesis is complex since the gene affects the cell in many different ways. The best known function of the EWS-FLI1 protein is that of an aberrant transcription factor [10]. Its structure consists of an N-terminal EWS transcriptional activation domain and a C-terminal FLI1 DNA-binding domain. Disruption of either domain impairs the transformation activity of the protein $[11,12]$.

EWS-FLI1 possesses the winged helix-turn-helix motif of the ETS family of transcription factors, and EWS-FLI1 recognizes the same consensus DNA binding sequence as FLI1. However, because of aberrant protein-protein interactions, in large part due to the EWS portion of the molecule, EWSFLI1 has an altered profile of gene regulation. It is relevant to note that FLI1 can act as an oncogene in its own right. Fli1 (Friend leukemia virus integration) was originally identified as the gene activated by insertion of the Friend murine leukemia virus (MuLV) [13]. It has the capacity to transform murine cells and is responsible for the development of 
erythroleukemias in mice [14]. It is presumably the fusion of the N-terminal moiety of EWS that confers the ability to induce Ewing's sarcoma in human cells.

Acting as either a transcriptional activator or repressor, EWS-FLI1 regulates a number of important target genes. EWS-FLI1 has been found to upregulate genes that promote cell survival and proliferation, including IGF1 [15], Myc [16], TOPK [17], NKX2.2 [18], ID2 [19], DAX1 [20], GLI1 [21], EZH2 [22], MK-STYX [23], and PLD2 [24]. EWS-FLI1 concomitantly represses genes that induce cell cycle arrest and apoptosis, including TGFB2 [25], p21 [26], p57kip [27], and IGFBP3 [28]. Interestingly, EWS-FLI1 also upregulates genes that are involved in cell differentiation such as SOX2 [29] and EZH2 [22].

While progress has been made in characterizing the downstream targets of EWS-FLI1, the properties of EWSFLI1 may not be fully explained on the basis of its activity as a transcription factor. There are other properties of EWS-FLI1 that have not been studied quite so extensively. These include RNA binding, RNA splicing and protein-protein interactions [30-37]. Furthermore, it has become increasingly clear that the cellular context has a large bearing upon the transformation process. Early experiments with EWS-FLI1 in NIH3T3 cells were somewhat artificial in the sense that these cells were immortalized murine fibroblasts. Paradoxically, in normal murine and human fibroblasts, EWS-FLI1 by itself does not transform cells; instead, it results in cell cycle arrest $[38,39]$. These findings suggest the possibility that EWSFLI1 may have complex antagonistic effects on different types of cells and underscore the importance of identifying the correct cell of origin for the disease.

\section{Primitive Neuroectodermal Features of Ewing's Sarcoma}

The origin of Ewing's sarcoma is a controversial topic [7]. Histologically, Ewing's sarcoma has a certain resemblance to primitive neuroectodermal cells, and it was once widely believed that the tumor arose from such cells [40, 41]. Early neural markers, such as neuron-specific enolase (NSE) and S-100, are present in some tumors [42, 43]. Ultrastructural features, such as neurosecretory granules, can also be observed with electron microscopy in some cases as well [41, 43]. Laboratory experiments have demonstrated that Ewing's sarcoma cells can be induced to differentiate towards the neural lineage in vitro and acquire neuritic processes under appropriate stimulation [44]. Conversely, introduction of EWS-FLI1 into neuroblastoma cell lines has been shown to make the cells less differentiated and acquire characteristics of Ewing's sarcoma [45].

In spite of considerable data pointing to a neuroectodermal origin, some doubt has been cast upon this idea. The neural markers seen in Ewing's sarcoma are present only in a minority of cases, and even in such tumors, they occur sporadically in cells. More importantly, EWS-FLI1 has been shown to induce neuroectodermal differentiation and upregulate a number of genes associated with early neural differentiation $[46,47]$. The findings raise the possibility that the neuroectodermal characteristics of Ewing's sarcoma might be a direct result of EWS-FLI1 expression and not necessarily the cell of origin.

\section{Mesenchymal Stem Cells}

An alternative hypothesis is that Ewing's sarcoma derives from a mesenchymal stem cell. This addresses one simple but nagging question regarding the neuroectodermal theory of histogenesis, namely, whether bone normally contains primitive neuroectodermal cells. While cranial bones develop from mesenchymal condensation of neuroectoderm [48], the long bones of the limbs originate from mesoderm [49], and there may not normally be primitive neuroectodermal cells in bone. Since most cases of Ewing's sarcoma arise in bone, it seems plausible that the cell of origin ought to be a normal resident of bone. It may be significant to note that mesenchymal cells in bone marrow can exhibit some characteristics of neuroectodermal cells $[48,50]$. The cells sometimes express neural markers spontaneously, including S-100 and neurofilament M [50]. They can also be induced in vitro to differentiate towards the neural lineage $[51,52]$.

A fundamental problem with the mesenchymal hypothesis is that one must define what exactly constitutes a "mesenchymal stem cell." The term is widely used in scientific literature, and yet the existence of the cell has not been rigorously proven or defined [53]. The term MSC usually implies a bone marrow-derived cell that has the capacity to differentiate towards various mesodermal lineages, such as osteoblasts, chondrocytes, and adipocytes. In order to satisfy the criteria for being a stem cell, it must be shown to be (1) self-renewing and (2) able to generate different tissue types in vivo from a single cell. This has proven to be a daunting task, and perhaps as a result, the putative MSC has yet to be defined in terms of a reliable set of cell surface markers that would aid greatly in consistent extraction of the cells [54]. Cells considered to be MSCs have been reported to be positive for a number of markers, including Sca-1, Stro-1, SH2, SH3, SH4, CD29, CD44, CD90, and CD105, but none of these are entirely specific for MSCs [53-58].

Most preparations of MSCs are heterogeneous [59]. Bone marrow extracts are typically plated on plastic dishes, and the adherent cells are enriched for cells that have some of the desired properties of MSCs. However, the cultures contain a mixture of cells, including hematopoietic stem cells, endothelial cells, osteoblasts, and other cells. The cells can be stimulated to undergo differentiation towards osteoblastic, chondroblastic, and adipocytic lineages in vitro under appropriate culture conditions. One notes though that unless cultures are derived from single cell colonies, it is possible that different cell subpopulations differentiate into the various lineages, and it is therefore not accurate to call the cells MSCs. Furthermore, expression of in vitro markers of differentiation does not equate to the capacity for in vivo differentiation [53]. For these reasons, some authors prefer to call these cell preparations by less presumptive terms, such as "bone marrow stromal cells" as opposed to MSCs.

There are data to support the idea that there is more than one type of pluripotential nonhematopoietic cell in bone marrow. Colter et al. showed that within the population of 
plastic adherent cells, there are at least two distinct subgroups which could be separated on the basis of size and cell surface markers [60]. A small, round cell with rapid doubling time seems to predominate at early passage whereas a larger spindle cell tends to divide more rapidly at later times. The latter cell type may be an osteoblast precursor and perhaps less pluripotential since it spontaneously expresses alkaline phosphatase, an osteoblast marker.

Jiang et al. showed that bone marrow contains a small population of pluripotential cells, which they termed "marrow-associated progenitor cells" (MAPCs) [61]. The cells represent a subset of the bone marrow cells that adhere to plastic. The cells were found to have clonogenic self-renewal capabilities. Furthermore, when injected into blastocysts, the cells developed into many different tissues, including those derived from ectoderm, endoderm, and mesoderm. These cells may be distinct from the majority of cells typically termed MSCs since their cell surface markers appear to be different from most of the plastic-adherent cells extracted from bone marrow.

At the present time, it should be recognized that our understanding of the "mesenchymal stem cell" is far from complete. The exact nature and composition of the majority of plastic-adherent bone marrow cells is still in question. The very primitive stem cell described by Jiang et al. [61] may be similar to the stem cells found in other tissues [62] and may not represent the more prevalent bone marrow stromal cell. Within the latter group of cells, there may be multiple subpopulations of cell types with varying pluripotential capabilities for in vivo differentiation, analogous to hematopoietic cells [54].

\section{MSC Characteristics of Ewing's Sarcoma}

There are several lines of evidence that support the notion that Ewing's sarcoma is derived from an MSC-like cell (accepting for the moment the concept of the MSC). Using Ewing's sarcoma cell lines, researchers have knocked down the expression of EWS-FLI1 and shown that the cells have some capacity for in vitro differentiation towards chondroblastic, osteoblastic, and adipocytic lineages [63]. Several groups have demonstrated that expression of EWSFLI1 in murine MSCs resulted in transformation of the cells, and when these cells were implanted into mice, sarcomas formed $[64,65]$. The tumors shared some characteristics with Ewing's sarcoma, including cell surface markers and cell morphology. In a related set of experiments, expression of EWS-FLI1 in the pluripotential murine cell line C3H10T1/2 inhibited the cells ability to differentiate into osteoblasts and adipocytes while upregulating neural genes [66]. When injected into mice, these cells formed metastatic sarcomas.

In contrast to the mouse studies, human MSCs that were infected with a retrovirus containing EWS-FLI1 failed to form tumors when injected into immunodeficient mice [67]. Although somewhat disappointing, the results are not at all surprising since it has been reported that transformation of normal human mesenchymal cells requires multiple mutations [68]. Murine mesenchymal cells are more apt to transform spontaneously in culture and form sarcomas [69].
It is worthwhile to note that human cells expressing EWSFLI1 take on a rounded morphology and express some of the neuroectodermal markers seen in Ewing's sarcoma $[67,70]$. Furthermore, the expression of CD99, a relatively specific marker for Ewing's sarcoma, was found to be present at a low level in MSCs and upregulated by EWS-FLI1 [67]. This addressed a previously raised concern against the theory of an MSC origin of Ewing's sarcoma regarding expression of CD99 in MSCs [71].

The relationship between Ewing's sarcoma and human mesodermal cells has been strengthened further in other experiments. Ewing's sarcoma cell lines with knockdown of EWS-FLI1 have a transcription profile similar to that of the human fetal fibroblast cell line IMR-90 [75]. Similarly, Tirode et al. showed that in an EWS-FLI1 knock-down experiment, the gene expression profile of the cells began to converge upon that of mesenchymal stem cells [63]. This was statistically significant for the subset of genes that were upregulated or downregulated by EWS-FLI1, but it is noteworthy that the overall pattern of gene expression appeared to have distinct differences between MSCs and Ewing's sarcoma cells with EWS-FLI1 knockdown. Comparison of cell surface markers has also shown some similarities and differences between MSCs and Ewing's sarcoma (Table 1). A complementary set of observations was made by Burns et al., who reported that late passage human mesenchymal cells that spontaneously transformed after introduction of telomerase (hMSC-TERT20) took on an immunohistochemical profile that was reminiscent of Ewing's sarcoma, namely, CD99+, vimentin+, CD45-, cytokeratin-, and desmin[72]. Finally, in a meta-analysis of studies on genes affected by EWS-FLI1, a "core EWS-FLI1 transcriptional signature" was identified which shared similarities with published mesenchymal stem cell data [76]. While these collective data are supportive of a mesenchymal origin of Ewing's sarcoma, it is clear that simple knock-down of EWS-FLI1 in tumor cells does not cause them to revert to a normal mesenchymal cell. Other changes have accumulated in the cells that make them distinctly different from recognizable normal cells.

\section{Knock-in and Transgenic Mouse Models}

The importance of cellular context has been highlighted in recent mouse models. Several knock-in and transgenic mice expressing EWS-FLI1 have now been created. In all of these models, a strategy of conditional expression has been employed to avoid lethal effects of EWS-FLI1. Constitutive expression of EWS-FLI1 protein in embryonic stem cells causes cell death [77], and mice with expression of EWSFLI1 in the whole body have an embryonic lethal phenotype $[78,79]$. To circumvent this problem, investigators have used cre-loxP recombination to achieve conditional expression of the protein in vivo. Tissue-specific cre transgenic mice are crossed to the EWS-FLI1 mice to restrict expression of the protein to certain tissues or cells.

Torchia et al. developed a knock-in EWS-FLI1 mouse at the Rosa26 locus [78]. When EWS-FLI1 was expressed by use of the Mx1-cre mouse, myeloid/erythroid leukemias developed. These malignancies were similar to but distinct 
TABLE 1: Cell surface marker analysis of mesenchymal cells and Ewing's sarcoma.

\begin{tabular}{|c|c|c|c|c|c|}
\hline Marker & Mes. Cells & Mes. Cells + EWS-FLI1 & ESFT & $\begin{array}{c}\text { ESFT + EWS-FLI1 } \\
\text { knockdown }\end{array}$ & Refs. \\
\hline $\mathrm{CD} 10$ & + & - & - & & [70] \\
\hline $\mathrm{CD} 13$ & + & - & - & & [70] \\
\hline $\mathrm{CD} 29$ & + & & - & + & {$[63]$} \\
\hline CD44 & + & + & - & + & {$[57,63,70]$} \\
\hline $\begin{array}{l}\text { CD45 (leukocyte } \\
\text { common antigen) }\end{array}$ & - & & - & & {$[57,72]$} \\
\hline CD54 & $\mp$ & + & $\mp$ & + & {$[63,70]$} \\
\hline CD59 & + & & - & + & {$[63]$} \\
\hline CD73 & + & & - & + & {$[63]$} \\
\hline CD99 (MIC2) & $\mp$ & + & ++ & + & {$[67,70,71,73]$} \\
\hline CD105 & + & + & $\mp$ & + & {$[57,63,70]$} \\
\hline CD 117 (c-kit) & - & + & + & & {$[70]$} \\
\hline CD166 & + & + & + & + & {$[63,70]$} \\
\hline $\mathrm{CD} 271$ & - & + & + & & [70] \\
\hline Vimentin & + & & + & & [72] \\
\hline Caveolin-1 & + & & + & & {$[73,74]$} \\
\hline Desmin & - & & - & & {$[72]$} \\
\hline Cytokeratin & - & & - & & {$[72]$} \\
\hline
\end{tabular}

Mes. cells: human bone marrow-derived mesenchymal cells.

ESFT: Ewing's sarcoma family of tumors.

₹ indicates weak or variable staining.

from the erythroleukemias induced by F-MuLV. The Mx1cre mouse expresses cre recombinase in the liver, spleen, bone marrow, and lymphoid tissues after induction with alpha/beta interferon or polyinosinic* poly $(\mathrm{C})$ (pIpC) [80]. While the original strategy was to target expression to bone marrow progenitor cells, it is likely that the gene was preferentially expressed in hematopoietic precursors, thereby producing leukemias.

Codrington et al. developed an interesting Ews-ERG mouse, which possesses an inverted ERG cassette flanked by loxP sites in intron 8 of the Ews gene, to simulate the $t(21 ; 22)$ translocation that produces the EWS-ERG fusion [81]. They obtained $\mathrm{T}$ cell lymphomas in all mice but this may have been due to their choice of the Rag1-cre mouse, which expresses cre recombinase in lymphocytes only [82].

Although it may be somewhat surprising that leukemias and lymphomas were found in the previous two mouse models, there is reason to believe that this has clinical relevance to human disease. While it was once believed that EWS-FLI1 was specific to Ewing's sarcoma, it is now known that EWS-FLI1 and related EWS-ETS fusions occur sporadically in other malignancies, including leukemias [8387] and biphenotypic tumors, which have features of both myogenic and neuroectodermal differentiation [88]. These cases indicate that EWS-FLI1 can potentially contribute to neoplastic growth outside of the typical cellular context in which Ewing's sarcoma arises. Quite interestingly, the CD99 marker, which is commonly used for immunohistochemical testing for Ewing's sarcoma, is actually considered a normal leukocyte marker and is uniformly expressed on thymocytes [89].

In a different transgenic mouse model of EWS-FLI1, conditional expression of the fusion protein was achieved in the mesoderm-derived tissues of the limbs by crossing to the Prx1-cre mouse, which expresses cre in the primitive mesenchyme of the early limb bud [79]. Part of the rationale behind this cross was to spare the hematopoietic cells and thereby increase the likelihood of inducing sarcomas. Limb shortening, muscle atrophy, osseous dysplasia, and other developmental abnormalities were observed, reinforcing the idea that EWS-FLI1 impairs growth and differentiation of cells. However, in this model, sarcomas did not spontaneously form unless the p53 gene was simultaneously mutated [79]. In contrast to the more differentiated osteosarcomas that formed without EWS-FLI1 in mice with conditional mutation of $p 53$ induced by Prx1-cre, the tumors that arose with EWS-FLI1 were undifferentiated sarcomas, similar to Ewing's sarcoma.

Although concern has been raised regarding the validity of mouse models for Ewing's sarcoma [76], the transgenic mouse model employing Prxl-cre does underscore one important feature of Ewing's sarcoma, namely, that EWSFLI1 alone does not appear to be sufficient to confer sarcomatous change in an in vivo setting. Similar to the results obtained with human MSCs expressing EWS-FLI1, additional cooperating mutations seem to be required for transformation to occur. The nature and timing of these mutations may be an interesting subject for future research 


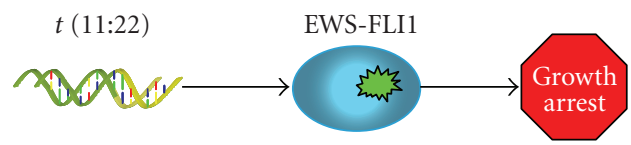

(a)

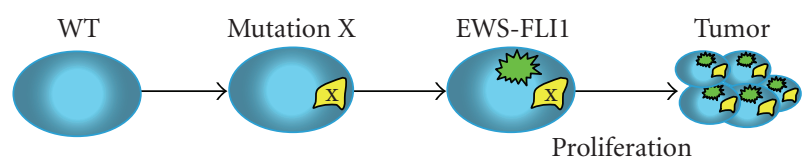

(b)

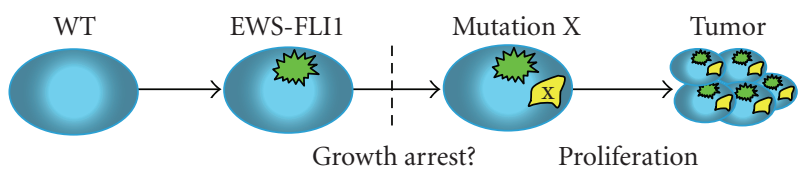

(c)

Figure 1: Cooperative mutations in the development of Ewing's sarcoma. (a) A $t(11 ; 22)$ reciprocal translocation produces the EWS-FLI1 gene, but this tends to cause growth arrest in normal cells. (b) A mutation randomly occurring prior to the $t(11 ; 22)$ translocation might cooperate with EWS-FLI1 to permit escape from growth arrest (or even promote cell proliferation) and subsequent transformation to Ewing's sarcoma. (c) The cooperative mutation may occur after the $\mathrm{t}(11 ; 22)$ translocation; this would necessarily imply a mechanism for continued cell growth after EWSFLI1 is expressed.

(Figure 1). The limb shortening of transgenic mice [79] and experimental cell culture work of various groups $[39,50]$ suggest that EWS-FLI1 impairs growth of normal cells, and this may be problematic in envisioning a pathway for neoplastic transformation to occur. While many have postulated that mutations occur after the $\mathrm{t}(11 ; 22)$ translocation event, it is also possible that EWS-FLI1 transforms a cell that carries a silent but critical mutation in another gene.

The requirement for additional mutational events has precedent in other mouse models. For example, in a knockin model of alveolar rhabdomyosarcoma, mutations in Ink4A/Arf or Trp53 were needed for the Pax3;Fkhr fusion to transform cells effectively [90]. Interestingly, conditional expression in the correct cell of origin-postnatal differentiating myofibers-was also found to be important for rhabdomyosarcomas to develop in this particular model. TRP53, however, is unlikely to be the critical cooperating mutated gene in Ewing's sarcoma since TRP53 mutations are found only in about 10\% of Ewing's sarcoma cases [91].

\section{Conclusions}

Identification of the correct cell of origin is crucial to the understanding of Ewing's sarcoma. While there has been steadily accumulating evidence that the cell of origin may be a primitive pluripotential cell that resides in bone marrow, one cannot state unequivocally that it is either a neuroectodermal cell or a "mesenchymal stem cell." One important simple reason for this is that the concept of the MSC has yet to be rigorously defined, and the term is used somewhat loosely in the scientific literature. MSCs, as they are typically prepared, are composed of a mixture of different cells, and some of these do exhibit neuroectodermal properties. It is possible that a subpopulation of the pluripotential cells in bone marrow is especially vulnerable to the transforming effects of EWS-FLII.

Even if the correct cell of origin can be identified with precision, much has yet to be learned. Expression of EWSFLI1 alone is not sufficient for transformation of normal human cells. Instead, EWS-FLI1 tends to cause growth arrest. In the early excitement over the discovery of EWS-FLI1, researchers focused largely upon downstream transcriptional targets. Current research suggests that there may be critically important cooperating mutations that work in concert with EWS-FLI1. The "cell of origin" concept may be a simplistic one in that it might not be a normal cell from which Ewing's sarcoma arises. EWS-FLI1 may be fully transforming only in a mutated cell that possesses additional genetic alterations. The tumor that eventually arises, whether it is a conventional Ewing's sarcoma, an unusual variant, a biphenotypic tumor, lymphoblastic leukemia, or some other disease, will probably depend upon an interplay between the cellular background in which it arises and the set of cooperating mutations that occur.

\section{References}

[1] J. L. Ordóñez, D. Osuna, D. Herrero, E. de Álava, and J. MadozGúrpide, "Advances in Ewing's sarcoma research: where are we now and what lies ahead?" Cancer Research, vol. 69, no. 18, pp. 7140-7150, 2009.

[2] M. E. Nesbit Jr., E. A. Gehan, E. O. Burgert Jr. et al., "Multimodal therapy for the management of primary, nonmetastatic Ewing's Sarcoma of bone: a long-term follow-up of the first intergroup study," Journal of Clinical Oncology, vol. 8, no. 10, pp. 1664-1674, 1990.

[3] H. E. Grier, M. D. Krailo, N. J. Tarbell et al., "Addition of ifosfamide and etoposide to standard chemotherapy for Ewing's sarcoma and primitive neuroectodermal tumor of bone," New England Journal of Medicine, vol. 348, no. 8, pp. 694-701, 2003.

[4] C. Rodriguez-Galindo, C. A. Billups, L. E. Kun et al., "Survival after recurrence of ewing tumors: the St. Jude children's research hospital experience, 1979-1999," Cancer, vol. 94, no. 2, pp. 561-569, 2002.

[5] J. Ban, C. Siligan, M. Kreppel, D. Aryee, and H. Kovar, "EWSFLI1 in Ewing's sarcoma: real targets and collateral damage," Advances in Experimental Medicine and Biology, vol. 587, pp. 41-52, 2006.

[6] N. Riggi and I. Stamenkovic, "The Biology of Ewing sarcoma," Cancer Letters, vol. 254, no. 1, pp. 1-10, 2007.

[7] H. Kovar, "Context matters: the hen or egg problem in Ewing's sarcoma," Seminars in Cancer Biology, vol. 15, no. 3, pp. 189196, 2005.

[8] J. A. Toretsky, Y. Connell, L. Neckers, and N. K. Bhat, "Inhibition of EWS-FLI-1 fusion protein with antisense oligodeoxynucleotides," Journal of Neuro-Oncology, vol. 31, no. 1-2, pp. 9-16, 1997.

[9] K. Tanaka, T. Iwakuma, K. Harimaya, H. Sato, and Y. Iwamoto, "EWS-Fli1 antisense oligodeoxynucleotide inhibits proliferation of human Ewing's sarcoma and primitive neuroectodermal tumor cells," Journal of Clinical Investigation, vol. 99, no. 2, pp. 239-247, 1997. 
[10] A. Üren, O. Tcherkasskaya, and J. A. Toretsky, "Recombinant EWS-FLI1 oncoprotein activates transcription," Biochemistry, vol. 43, no. 42, pp. 13579-13589, 2004.

[11] W. A. May, M. L. Gishizky, S. L. Lessnick et al., "Ewing sarcoma 11;22 translocation produces a chimeric transcription factor that requires the DNA-binding domain encoded by FLI1 for transformation," Proceedings of the National Academy of Sciences of the United States of America, vol. 90, no. 12, pp. 5752-5756, 1993.

[12] S. L. Lessnick, B. S. Braun, C. T. Denny, and W. A. May, "Multiple domains mediate transformation by the Ewing's sarcoma EWS/FLI-1 fusion gene," Oncogene, vol. 10, no. 3, pp. 423-431, 1995.

[13] Y. Ben-David, E. B. Giddens, and A. Bernstein, "Identification and mapping of a common proviral integration site Fli-1 in erythroleukemia cells induced by Friend murine leukemia virus," Proceedings of the National Academy of Sciences of the United States of America, vol. 87, no. 4, pp. 1332-1336, 1990.

[14] A. H. L. Truong and Y. Ben-David, "The role of Fli-1 in normal cell function and malignant transformation," Oncogene, vol. 19, no. 55, pp. 6482-6489, 2000.

[15] L. Cironi, N. Riggi, P. Provero et al., "IGF1 is a common target gene of Ewing's sarcoma fusion proteins in mesenchymal progenitor cells," PLoS One, vol. 3, no. 7, Article ID e2634, 2008.

[16] M. R. Sollazzo, M. S. Benassi, G. Magagnoli et al., "Increased cmyc oncogene expression in Ewing's sarcoma: correlation with Ki67 proliferation index," Tumori, vol. 85, no. 3, pp. 167-173, 1999.

[17] D. Herrero-Martín, D. Osuna, J. L. Ordóñez et al., "Stable interference of EWS-FLI1 in an Ewing sarcoma cell line impairs IGF-1/IGF-1R signalling and reveals TOPK as a new target," British Journal of Cancer, vol. 101, no. 1, pp. 80-90, 2009.

[18] R. Smith, L. A. Owen, D. J. Trem et al., "Expression profiling of EWS/FLI identifies NKX2.2 as a critical target gene in Ewing's sarcoma," Cancer Cell, vol. 9, no. 5, pp. 405-416, 2006.

[19] M. Fukuma, H. Okita, J.-I. Hata, and A. Umezawa, "Upregulation of Id2, an oncogenic helix-loop-helix protein, is mediated by the chimeric EWS/ets protein in Ewing sarcoma," Oncogene, vol. 22 , no. 1, pp. 1-9, 2003.

[20] E. García-Aragoncillo, J. Carrillo, E. Lalli et al., "DAX1, a direct target of EWS/FLI1 oncoprotein, is a principal regulator of cell-cycle progression in Ewing's tumor cells," Oncogene, vol. 27, no. 46, pp. 6034-6043, 2008.

[21] J. P. Zwerner, J. Joo, K. L. Warner et al., "The EWS/FLI1 oncogenic transcription factor deregulates GLI1," Oncogene, vol. 27, no. 23, pp. 3282-3291, 2008.

[22] G. H. S. Richter, S. Plehm, A. Fasan et al., "EZH2 is a mediator of EWS/FLI1 driven tumor growth and metastasis blocking endothelial and neuro-ectodermal differentiation," Proceedings of the National Academy of Sciences of the United States of America, vol. 106, no. 13, pp. 5324-5329, 2009.

[23] C. Siligan, J. Ban, R. Bachmaier et al., "EWS-FLI1 target genes recovered from Ewing's sarcoma chromatin," Oncogene, vol. 24, no. 15, pp. 2512-2524, 2005.

[24] R. Kikuchi, M. Murakami, S. Sobue et al., "Ewing's sarcoma fusion protein, EWS/Fli-1 and Fli-1 protein induce PLD2 but not PLD1 gene expression by binding to an ETS domain of 5' promoter," Oncogene, vol. 26, no. 12, pp. 1802-1810, 2007.

[25] K.-B. Hahm, K. Cho, C. Lee et al., "Repression of the gene encoding the TGF- $\beta$ type II receptor is a major target of the EWS-FLI1 oncoprotein," Nature Genetics, vol. 23, no. 2, pp. 222-227, 1999.
[26] F. Nakatani, K. Tanaka, R. Sakimura et al., "Identification of p21WAF1/CIP1 as a direct target of EWS-Fli1 oncogenic fusion protein," Journal of Biological Chemistry, vol. 278, no. 17, pp. 15105-15115, 2003.

[27] L. Dauphinot, C. De Oliveira, T. Melot et al., "Analysis of the expression of cell cycle regulators in Ewing cell lines: EWS-FLI1 modulates p57KIP2 and c-Myc expression," Oncogene, vol. 20, no. 25, pp. 3258-3265, 2001.

[28] A. Prieur, F. Tirode, P. Cohen, and O. Delattre, "EWS/FLI-1 silencing and gene profiling of Ewing cells reveal downstream oncogenic pathways and a crucial role for repression of insulin-like growth factor binding protein 3," Molecular and Cellular Biology, vol. 24, no. 16, pp. 7275-7283, 2004.

[29] N. Riggi, M.-L. Suvà, C. De Vito et al., "EWS-FLI-1 modulates miRNA145 and SOX2 expression to initiate mesenchymal stem cell reprogramming toward Ewing sarcoma cancer stem cells," Genes and Development, vol. 24, no. 9, pp. 916-932, 2010.

[30] L. Yang, H. A. Chansky, and D. D. Hickstein, "EWS.Fli1 fusion protein interacts with hyperphosphorylated RNA polymerase II and interferes with serine-arginine proteinmediated RNA splicing," Journal of Biological Chemistry, vol. 275, no. 48, pp. 37612-37618, 2000.

[31] J. A. Toretsky, V. Erkizan, A. Levenson et al., "Oncoprotein EWS-FLI1 activity is enhanced by RNA helicase A," Cancer Research, vol. 66, no. 11, pp. 5574-5581, 2006.

[32] H. A. Chansky, M. Hu, D. D. Hickstein, and L. Yang, "Oncogenic TLS/ERG and EWS/Fli-1 fusion proteins inhibit RNA splicing mediated by YB-1 protein," Cancer Research, vol. 61, no. 9, pp. 3586-3590, 2001.

[33] D. M. Gascoyne, G. R. Thomas, and D. S. Latchman, "The effects of Brn-3a on neuronal differentiation and apoptosis are differentially modulated by EWS and its oncogenic derivative EWS/Fli-1," Oncogene, vol. 23, no. 21, pp. 3830-3840, 2004.

[34] S. Kim, C. T. Denny, and R. Wisdom, "Cooperative DNA binding with AP-1 proteins is required for transformation by EWS-Ets fusion proteins," Molecular and Cellular Biology, vol. 26, no. 7, pp. 2467-2478, 2006.

[35] R. Petermann, B. M. Mossier, D. N. T. Aryee, V. Khazak, E. A. Golemis, and H. Kovar, "Oncogenic EWS-Fli1 interacts with hsRPB7, a subunit of human RNA polymerase II," Oncogene, vol. 17, no. 5, pp. 603-610, 1998.

[36] L. Spahn, R. Petermann, C. Siligan, J. A. Schmid, D. N. T. Aryee, and H. Kovar, "Interaction of the EWS NH2 terminus with BARD1 links the Ewing's sarcoma gene to a common tumor suppressor pathway," Cancer Research, vol. 62, no. 16, pp. 4583-4587, 2002.

[37] D. K. Watson, L. Robinson, D. R. Hodge, I. Kola, T. S. Papas, and A. Seth, "FLI1 and EWS-FLI1 function as ternary complex factors and ELK1 and SAP1a function as ternary and quaternary complex factors on the Egrl promoter serum response elements," Oncogene, vol. 14, no. 2, pp. 213-221, 1997.

[38] B. Deneen and C. T. Denny, "Loss of p16 pathways stabilizes EWS/FLI1 expression and complements EWS/FLI1 mediated transformation," Oncogene, vol. 20, no. 46, pp. 6731-6741, 2001.

[39] S. L. Lessnick, C. S. Dacwag, and T. R. Golub, “The Ewing's sarcoma oncoprotein EWS/FLI induces a p53-dependent growth arrest in primary human fibroblasts," Cancer Cell, vol. 1, no. 4, pp. 393-401, 2002.

[40] A. O. Cavazzana, J. S. Miser, J. Jefferson, and T. J. Triche, "Experimental evidence for a neural origin of Ewing's sarcoma of bone," American Journal of Pathology, vol. 127, no. 3, pp. 507-518, 1987. 
[41] C.-H. Suh, N. G. Ordóñez, J. Hicks, and B. Mackay, "Ultrastructure of the Ewing's sarcoma family of tumors," Ultrastructural Pathology, vol. 26, no. 2, pp. 67-76, 2002.

[42] W. J. Rettig, P. Garin-Chesa, and A. G. Huvos, "Ewing's sarcoma: new approaches to histogenesis and molecular plasticity," Laboratory Investigation, vol. 66, no. 2, pp. 133-137, 1992.

[43] A. Franchi, G. Pasquinelli, G. Cenacchi et al., "Immunohistochemical and ultrastructural investigation of neural differentiation in Ewing sarcoma/PNET of bone and soft tissues," Ultrastructural Pathology, vol. 25, no. 3, pp. 219-225, 2001.

[44] S. Navarro, M. Gonzalez-Devesa, A. Ferrandez-Izquierdo, T. J. Triche, and A. Llombart-Bosch, "Scanning electron microscopic evidence for neural differentiation in Ewing's sarcoma cell lines," Virchows Archiv A, vol. 416, no. 5, pp. 383391, 1990.

[45] C. J. Rorie, V. D. Thomas, P. Chen, H. H. Pierce, J. P. O’Bryan, and B. E. Weissman, "The Ews/Fli-1 fusion gene switches the differentiation program of neuroblastomas to Ewing sarcoma/peripheral primitive neuroectodermal tumors," Cancer Research, vol. 64, no. 4, pp. 1266-1277, 2004.

[46] M. A. Teitell, A. D. Thompson, P. H. B. Sorensen, H. Shimada, T. J. Triche, and C. T. Denny, "EWS/ETS fusion genes induce epithelial and neuroectodermal differentiation in NIH 3T3 fibroblasts," Laboratory Investigation, vol. 79, no. 12, pp. 15351543, 1999.

[47] S. Hu-Lieskovan, J. Zhang, L. Wu, H. Shimada, D. E. Schofield, and T. J. Triche, "EWS-FLI1 fusion protein up-regulates critical genes in neural crest development and is responsible for the observed phenotype of Ewing's family of tumors," Cancer Research, vol. 65, no. 11, pp. 4633-4644, 2005.

[48] Y. Takashima, T. Era, K. Nakao et al., "Neuroepithelial cells supply an initial transient wave of MSC differentiation," Cell, vol. 129, no. 7, pp. 1377-1388, 2007.

[49] B. R. Olsen, A. M. Reginato, and W. Wang, "Bone development," Annual Review of Cell and Developmental Biology, vol. 16, pp. 191-220, 2000.

[50] E. C. Torchia, S. Jaishankar, and S. J. Baker, "Ewing tumor fusion proteins block the differentiation of pluripotent marrow stromal cells," Cancer Research, vol. 63, no. 13, pp. 3464$3468,2003$.

[51] G. C. Kopen, D. J. Prockop, and D. G. Phinney, "Marrow stromal cells migrate throughout forebrain and cerebellum, and they differentiate into astrocytes after injection into neonatal mouse brains," Proceedings of the National Academy of Sciences of the United States of America, vol. 96, no. 19, pp. 10711-10716, 1999.

[52] D. Woodbury, E. J. Schwarz, D. J. Prockop, and I. B. Black, "Adult rat and human bone marrow stromal cells differentiate into neurons," Journal of Neuroscience Research, vol. 61, no. 4, pp. 364-370, 2000.

[53] P. Bianco, P. G. Robey, and P. J. Simmons, "Mesenchymal stem cells: revisiting history, concepts, and assays," Cell Stem Cell, vol. 2, no. 4, pp. 313-319, 2008.

[54] N. Beyer Nardi and L. da Silva Meirelles, "Mesenchymal stem cells: isolation, in vitro expansion and characterization," Handbook of Experimental Pharmacology, no. 174, pp. 249282, 2006.

[55] W. R. Otto and J. Rao, “Tomorrow's skeleton staff: mesenchymal stem cells and the repair of bone and cartilage," Cell Proliferation, vol. 37, no. 1, pp. 97-110, 2004.

[56] R. J. Deans and A. B. Moseley, "Mesenchymal stem cells: biology and potential clinical uses," Experimental Hematology, vol. 28 , no. 8 , pp. 875-884, 2000.
[57] T. A. Lodie, C. E. Blickarz, T. J. Devarakonda et al., "Systematic analysis of reportedly distinct populations of multipotent bone marrow-derived stem cells reveals a lack of distinction," Tissue Engineering, vol. 8, no. 5, pp. 739-751, 2002.

[58] F. Anjos-Afonso, E. K. Siapati, and D. Bonnet, "In vivo contribution of murine mesenchymal stem cells into multiple cell-types under minimal damage conditions," Journal of Cell Science, vol. 117, no. 23, pp. 5655-5664, 2004.

[59] D. J. Prockop, "Marrow stromal cells as stem cells for nonhematopoietic tissues," Science, vol. 276, no. 5309, pp. 7174, 1997.

[60] D. C. Colter, I. Sekiya, and D. J. Prockop, "Identification of a subpopulation of rapidly self-renewing and multipotential adult stem cells in colonies of human marrow stromal cells," Proceedings of the National Academy of Sciences of the United States of America, vol. 98, no. 14, pp. 7841-7845, 2001.

[61] Y. Jiang, B. N. Jahagirdar, R. L. Reinhardt et al., "Pluripotency of mesenchymal stem cells derived from adult marrow," Nature, vol. 418, no. 6893, pp. 41-49, 2002.

[62] A. P. Beltrami, D. Cesselli, N. Bergamin et al., "Multipotent cells can be generated in vitro from several adult human organs (heart, liver, and bone marrow)," Blood, vol. 110, no. 9, pp. 3438-3446, 2007.

[63] F. Tirode, K. Laud-Duval, A. Prieur, B. Delorme, P. Charbord, and O. Delattre, "Mesenchymal stem cell features of Ewing tumors," Cancer Cell, vol. 11, no. 5, pp. 421-429, 2007.

[64] Y. Castillero-Trejo, S. Eliazer, L. Xiang, J. A. Richardson, and R. L. Ilaria Jr., "Expression of the EWS/FLI-1 oncogene in murine primary bone-derived cells results in EWS/FLI-1-dependent, Ewing sarcoma-like tumors," Cancer Research, vol. 65, no. 19, pp. 8698-8705, 2005.

[65] N. Riggi, L. Cironi, P. Provero et al., "Development of Ewing's sarcoma from primary bone marrow-derived mesenchymal progenitor cells," Cancer Research, vol. 65, no. 24, pp. 1145911468, 2005.

[66] I. González, S. Vicent, E. de Alava, and F. Lecanda, "EWS/FLI1 oncoprotein subtypes impose different requirements for transformation and metastatic activity in a murine model," Journal of Molecular Medicine, vol. 85, no. 9, pp. 1015-1029, 2007.

[67] N. Riggi, M.-L. Suvà, D. Suvà et al., "EWS-FLI-1 expression triggers a ewing's sarcoma initiation program in primary human mesenchymal stem cells," Cancer Research, vol. 68, no. 7, pp. 2176-2185, 2008.

[68] J. M. Funes, M. Quintero, S. Henderson et al., "Transformation of human mesenchymal stem cells increases their dependency on oxidative phosphorylation for energy production," Proceedings of the National Academy of Sciences of the United States of America, vol. 104, no. 15, pp. 6223-6228, 2007.

[69] Y. F. Zhou, M. Bosch-Marce, H. Okuyama et al., "Spontaneous transformation of cultured mouse bone marrow-derived stromal cells," Cancer Research, vol. 66, no. 22, pp. 1084910854, 2006.

[70] Y. Miyagawa, H. Okita, H. Nakaijima et al., "Inducible expression of chimeric EWS/ETS proteins confers Ewing's family tumor-like phenotypes to human mesenchymal progenitor cells," Molecular and Cellular Biology, vol. 28, no. 7, pp. 21252137, 2008.

[71] H. Kovar and A. Bernard, "CD99-positive "Ewing's sarcoma" from mouse bone marrow-derived mesenchymal progenitor cells?" Cancer Research, vol. 66, no. 19, p. 9786, 2006.

[72] J. S. Burns, B. M. Abdallah, H. D. Shrøder, and M. Kassem, "The histopathology of a human mesenchymal stem cell experimental tumor model: support for an hMSC origin for 
Ewing's sarcoma?" Histology and Histopathology, vol. 23, no. 10, pp. 1229-1240, 2008.

[73] A. Llombart-Bosch, I. Machado, S. Navarro et al., "Histological heterogeneity of Ewing's sarcoma/PNET: an immunohistochemical analysis of 415 genetically confirmed cases with clinical support," Virchows Archiv, vol. 455, no. 5, pp. 397-411, 2009.

[74] O. M. Tirado, S. Mateo-Lozano, J. Villar et al., "Caveolin-1 (CAV1) is a target of EWS/FLI-1 and a key determinant of the oncogenic phenotype and tumorigenicity of Ewing's sarcoma cells," Cancer Research, vol. 66, no. 20, pp. 9937-9947, 2006.

[75] G. Potikyan, K. A. France, M. R. J. Carlson, J. Dong, S. F. Nelson, and C. T. Denny, "Genetically defined EWS/FLI1 model system suggests mesenchymal origin of Ewing's family tumors," Laboratory Investigation, vol. 88, no. 12, pp. 12911302, 2008.

[76] J. D. Hancock and S. L. Lessnick, "A transcriptional profiling meta-analysis reveals a core EWS-FLI gene expression signature," Cell Cycle, vol. 7, no. 2, pp. 250-256, 2008.

[77] E. J. Sohn, H. Li, K. Reidy, L. F. Beers, B. L. Christensen, and S. B. Lee, "EWS/FLI1 oncogene activates caspase 3 transcription and triggers apoptosis in vivo," Cancer Research, vol. 70, no. 3, pp. 1154-1163, 2010.

[78] E. C. Torchia, K. Boyd, J. E. Rehg, C. Qu, and S. J. Baker, "EWS/FLI-1 induces rapid onset of myeloid/erythroid leukemia in mice," Molecular and Cellular Biology, vol. 27, no. 22, pp. 7918-7934, 2007.

[79] P. P. Lin, M. K. Pandey, F. Jin et al., "EWS-FLI1 induces developmental abnormalities and accelerates sarcoma formation in a transgenic mouse model," Cancer Research, vol. 68, no. 21, pp. 8968-8975, 2008.

[80] R. Kuhn, F. Schwenk, M. Aguet, and K. Rajewsky, "Inducible gene targeting in mice," Science, vol. 269, no. 5229, pp. 14271429, 1995.

[81] R. Codrington, R. Pannell, A. Forster et al., "The Ews-ERG fusion protein can initiate neoplasia from lineage-committed haematopoietic cells," PLoS Biology, vol. 3, no. 8, article e242, 2005.

[82] M. P. McCormack, A. Forster, L. Drynan, R. Pannell, and T. H. Rabbitts, "The LMO2 T-cell oncogene is activated via chromosomal translocations or retroviral insertion during gene therapy but has no mandatory role in normal T-cell development," Molecular and Cellular Biology, vol. 23, no. 24, pp. 9003-9013, 2003.

[83] G. Jakovljević, M. Nakić, S. Rogošić et al., "Pre-B-cell acute lymphoblastic leukemia with bulk extramedullary disease and chromosome 22 (EWSR1) rearrangement masquerading as ewing sarcoma," Pediatric Blood and Cancer, vol. 54, no. 4, pp. 606-609, 2010.

[84] A. Martini, R. La Starza, H. Janssen et al., "Recurrent rearrangement of the Ewing's sarcoma gene, EWSR1, or its homologue, TAF15, with the transcription factor CIZ/NMP4 in acute leukemia," Cancer Research, vol. 62, no. 19, pp. 54085412, 2002.

[85] G. Marcucci, C. D. Baldus, A. S. Ruppert et al., "Overexpression of the ETS-related gene, ERG, predicts a worse outcome in acute myeloid leukemia with normal karyotype: a Cancer and Leukemia Group B study," Journal of Clinical Oncology, vol. 23, no. 36, pp. 9234-9242, 2005.

[86] J. M. Hawkins, J. M. Craig, L. M. Secker-Walker, H. G. Prentice, and A. B. Mehta, "Ewing's sarcoma $t(11 ; 22)$ in a case of acute nonlymphocytic leukemia," Cancer Genetics and Cytogenetics, vol. 55, no. 2, pp. 157-162, 1991.
[87] K. Haresh, N. Joshi, C. Gupta et al., "Granulocytic sarcoma masquerading as Ewing's sarcoma: a diagnostic dilemma," Journal of Cancer Research and Therapeutics, vol. 4, no. 3, pp. 137-139, 2008.

[88] P. H. B. Sorensen, H. Shimada, X. F. Liu, J. F. Lim, G. Thomas, and T. J. Triche, "Biphenotypic sarcomas with myogenic and neural differentiation express the Ewing's sarcoma EWS/FLI1 fusion gene," Cancer Research, vol. 55, no. 6, pp. 1385-1392, 1995.

[89] C. Aussel, G. Bernard, J.-P. Breittmayer, C. Pelassy, D. Zoccola, and A. Bernard, "Monoclonal antibodies directed against the E2 protein (MIC2 gene product) induce exposure of phosphatidylserine at the thymocyte cell surface," Biochemistry, vol. 32, no. 38, pp. 10096-10101, 1993.

[90] C. Keller, B. R. Arenkiel, C. M. Coffin, N. El-Bardeesy, R. A. DePinho, and M. R. Capecchi, "Alveolar rhabdomyosarcomas in conditional Pax3:Fkhr mice: cooperativity of Ink4a/ARF and Trp53 loss of function," Genes and Development, vol. 18, no. 21, pp. 2614-2626, 2004.

[91] H. Y. Huang, P. B. Illei, Z. Zhao et al., "Ewing sarcomas with p53 mutation or p16/p14ARF homozygous deletion: a highly lethal subset associated with poor chemoresponse," Journal of Clinical Oncology, vol. 23, no. 3, pp. 548-558, 2005. 


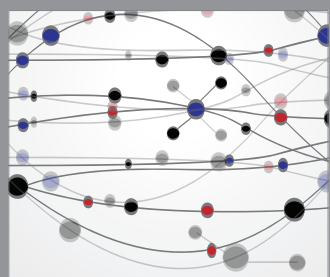

The Scientific World Journal
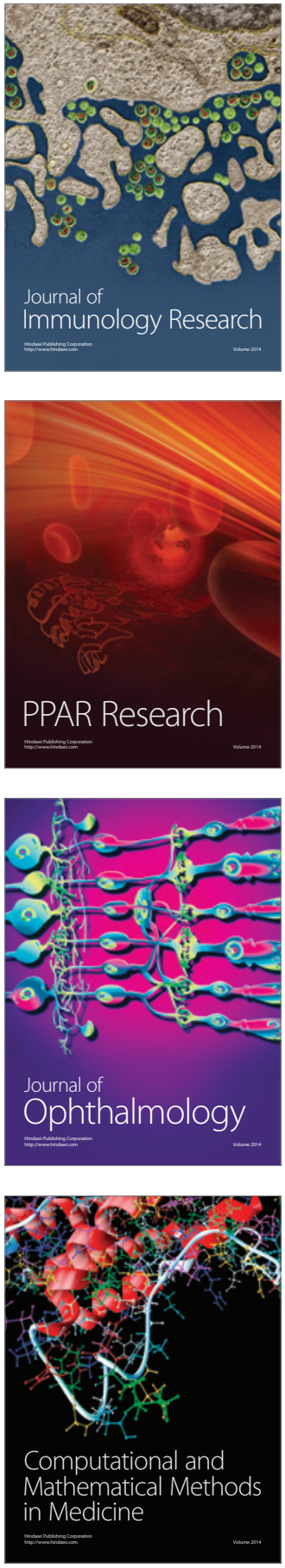

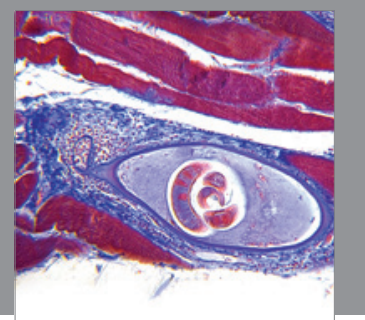

Gastroenterology

Research and Practice
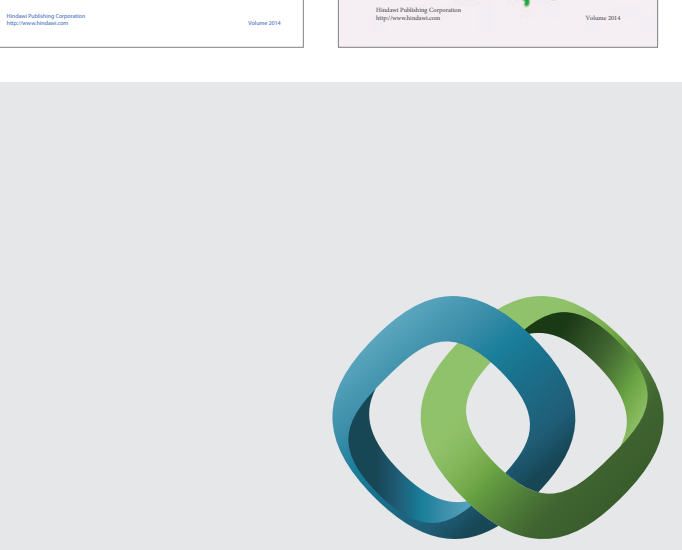

\section{Hindawi}

Submit your manuscripts at

http://www.hindawi.com
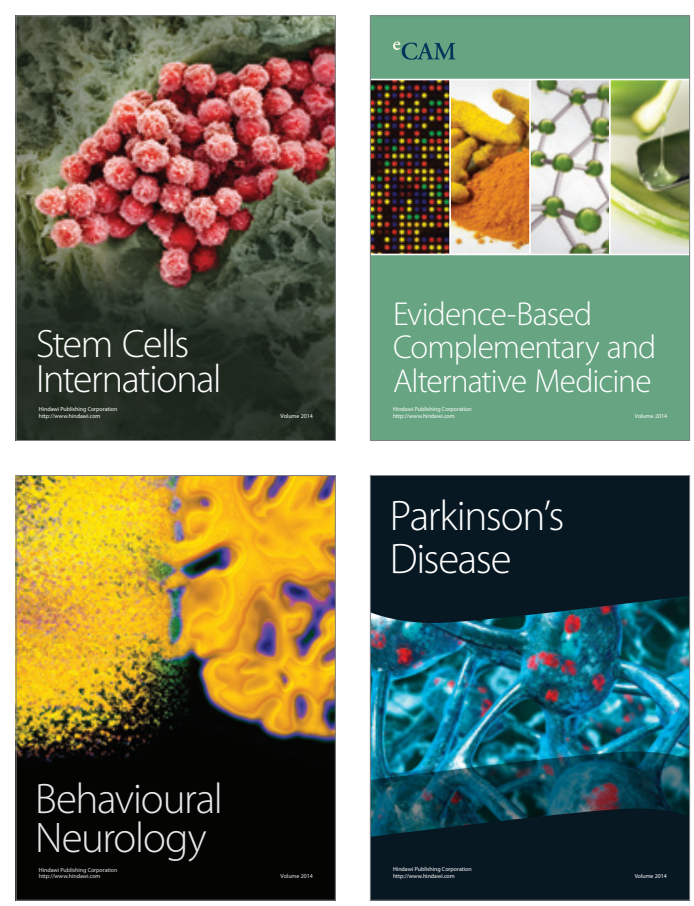

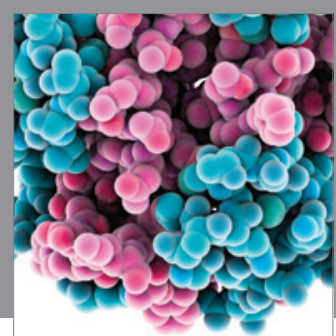

Journal of
Diabetes Research

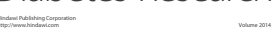

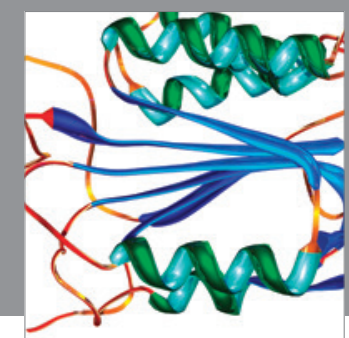

Disease Markers
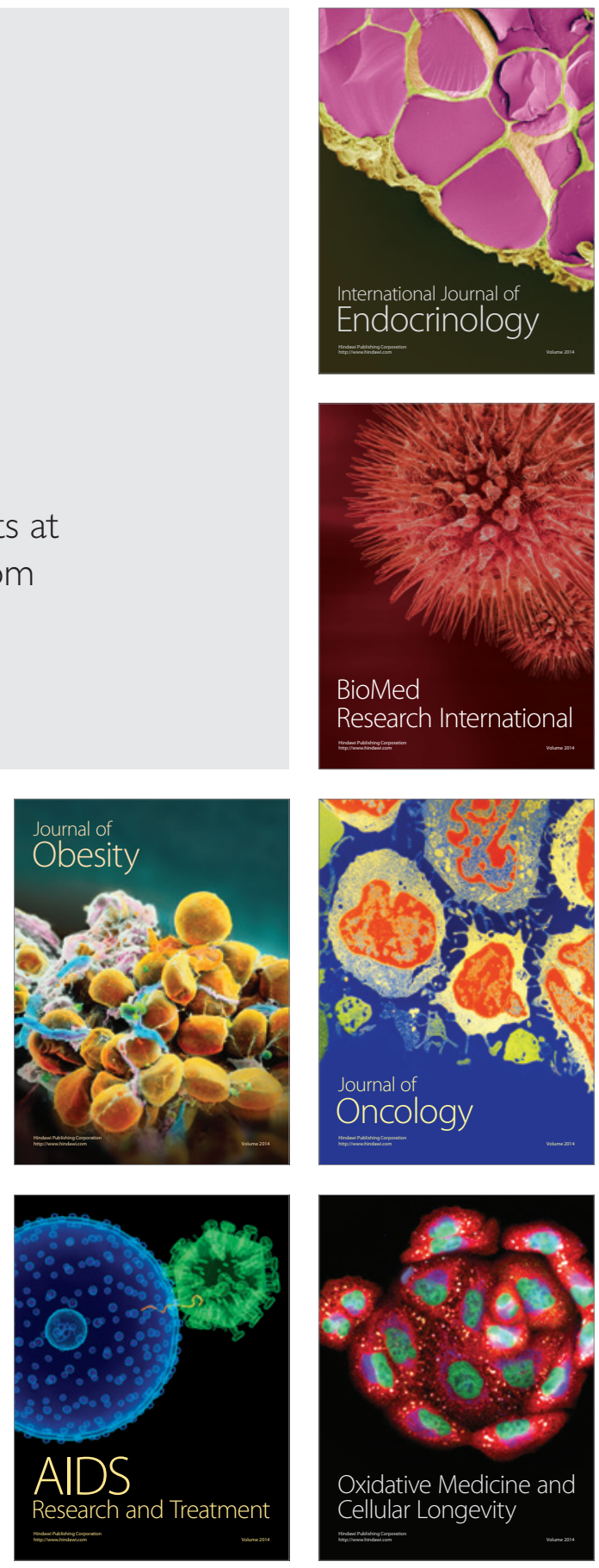